\title{
Dipyridamole decreases dialysis risk and improves survival in patients with pre-dialysis advanced chronic kidney disease
}

\author{
Ko-Lin Kuo ${ }^{1,2}$, Szu-Chun Hung ${ }^{1,2}$, Wei-Cheng Tseng ${ }^{3,4}$, Jia-Sin Liu ${ }^{5}$, Ming-Huang Lin ${ }^{6}$, \\ Chih-Cheng Hsu ${ }^{4,6,7}$ and Der-Cherng Tarng ${ }^{3,4,8}$ \\ ${ }^{1}$ Division of Nephrology, Taipei Tzu Chi Hospital, Buddhist Tzu Chi Medical Foundation, Hualien, Taiwan \\ ${ }^{2}$ School of Medicine, Tzu Chi University, Hualien, Taiwan \\ ${ }^{3}$ Division of Nephrology, Department of Medicine, Taipei Veterans General Hospital, Taipei, Taiwan \\ ${ }^{4}$ Institute of Clinical Medicine and Faculty of Medicine, National Yang-Ming University, Taipei, Taiwan \\ ${ }^{5}$ Department of Public Health, Kaohsiung Medical University, Kaohsiung, Taiwan \\ ${ }^{6}$ Institute of Population Health Sciences, National Health Research Institutes, Zhunan, Taiwan \\ ${ }^{7}$ Department of Health Services Administration, China Medical University, Taichung, Taiwan \\ ${ }^{8}$ Department and Institute of Physiology, National Yang-Ming University, Taipei, Taiwan \\ Correspondence to: Chih-Cheng Hsu, email: cch@nhri.org.tw \\ Der-Cherng Tarng, email: dctarng@vghtpe.gov.tw
}

Keywords: chronic kidney disease, dialysis, erythropoiesis-stimulating agent, dipyridamole, renin-angiotensin-aldosterone system blockade

Received: May 07, $2017 \quad$ Accepted: July 25, $2017 \quad$ Published: August 03, 2017

Copyright: Kuo et al. This is an open-access article distributed under the terms of the Creative Commons Attribution License 3.0 (CC BY 3.0), which permits unrestricted use, distribution, and reproduction in any medium, provided the original author and source are credited.

\section{ABSTRACT}

Introduction: Dipyridamole decreases proteinuria and improves renal function progression in patients with glomerular disease through its inhibition of platelet activation and enhanced nitric oxide expression. Few studies have evaluated the effects of dipyridamole on renal outcome or survival in CKD stage 5 patients who have not yet received dialysis (CKD 5 ND).

Materials and Methods: A prospective cohort study was conducted based on the Taiwan National Health Insurance Research Database. From January 1, 2000 to June 30, 2009, we enrolled 28,497 patients who had a serum creatinine $>6 \mathbf{m g}$ / $\mathrm{dL}$ and a hematocrit $<28 \%$ and who were treated with erythropoiesis-stimulating agents (ESAs). All patients were further divided into two groups with or without dipyridamole use within 90 days after starting ESA therapy. Patient followed-up took place until dialysis, death before initiation of dialysis or December 31, 2009. The primary outcomes were long-term dialysis and death before initiating dialysis.

Results: The dipyridamole users and nonusers groups included 7,746 and 20,751 patients, respectively. We found that 20,152 patients $(70.7 \%)$ required long-term dialysis and 5,697 patients $(20.0 \%)$ died before a progression to end-stage renal disease required dialysis. After propensity score-matching, dipyridamole users were associated with lower risks for long-term dialysis (adjusted HR, 0.96 ; 95\% CI, 0.930.99 ) and death (adjusted HR, 0.91; 95\% CI, 0.85-0.97) compared with nonusers.

Conclusions: Dipyridamole exhibited a protective effect in reducing the risk for long-term dialysis and death among CKD 5 ND patients. Randomized studies are needed to validate this association. 


\section{INTRODUCTION}

Chronic kidney disease (CKD) has profound impacts on public health and the economy [1]. Activation of the renin-angiotensin-aldosterone system (RAAS) and the production of growth factors and inflammatory mediators play pivotal roles in CKD progression [2-5]. Cumulative evidence strongly recommends RAAS blockade, primarily with an angiotensin converting enzyme inhibitor (ACEI) or an angiotensin II receptor blocker (ARB), as first-line antihypertensive agents for the treatment of CKD [6]. Inhibition of the RAAS not only delays the progression of CKD both in non-diabetic and diabetic stage 1-3 CKD patients but also in non-diabetic stage 4 CKD patients in randomized control trials [7-12]. Our previous study demonstrated that the use of RAAS blockade in patients with stage 5 CKD who had not yet received dialysis (CKD $5 \mathrm{ND}$ ) was associated with a lower risk for long-term dialysis [13]. Most patients eventually progress to end-stage renal disease (ESRD) even after the intensive use of RAAS blockade. Therefore, it is important to find another strategy to arrest CKD progression.

Dipyridamole stimulates nitric oxide action and inhibits platelet aggregation via the inhibition of phosphodiesterase and has an antioxidant effect [14-16]. A number of clinical studies have shown the reno-protective effects of dipyridamole monotherapy or combination therapy with ACEIs, antiplatelet agents or immunosuppressants in the treatment of early CKD in patients with diabetic kidney disease, IgA nephropathy, and membranoproliferative glomerulonephritis [17-19]. However, studies focusing on dipyridamole monotherapy or its interaction with RAAS blockade in patients with CKD 5 ND that have used hard end points, such as ESRD and mortality, are limited.

Based on Taiwan National Health Insurance (NHI) reimbursement regulations, CKD patients who have a serum creatinine concentration of $>6 \mathrm{mg} / \mathrm{dL}$ (approximately stage 5 CKD) and a hematocrit of $<28 \%$ could receive erythropoiesis stimulating agent (ESA) to keep a hematocrit concentration not exceeding 36\%. This policy provides a unique opportunity to evaluate a study cohort with advanced CKD. To extend the current knowledge about dipyridamole therapy to pre-dialysis advanced CKD, we conducted a nationwide, cohort study to evaluate the association between dipyridamole treatment and the risks of long-term dialysis or death and the interaction between dipyridamole treatment and RAAS blockade.

\section{RESULTS}

\section{Patient characteristics}

Figure 1 shows the flow chart for patient selection. The date of the initiation of ESA therapy for each patient was defined as the index date. After excluding those ineligible, we selected 28,497 individuals with CKD $5 \mathrm{ND}$ for further analysis. All patients were classified as dipyridamole users or nonusers within 90 days of the index date. Among this population, 7,746 (27.2\%) patients were dipyridamole users and 20,751 (72.8\%) were nonusers. The mean age of the dipyridamole users was 66 years, of whom $47.1 \%$ were male and $50.6 \%$ had diabetes mellitus (Table 1). Compared with the dipyridamole nonusers, the dipyridamole users were older, and less likely to visit nephrologists in the preceding 3 years. Because we expected dipyridamole users and nonusers to differ with respect to prognostic factors confound the outcome analyses, we used a propensity score-based matching to control residual confounding factors. For each dipyridamole user, we identified two nonusers from our selected cohort who has the most similar estimated propensity scores which were calculated from all the baseline characteristics in Table 1. The formula of propensity scores were also listed in Supplementary Table 1. We applied the nearest-neighbor algorithm was to construct matched pairs, assuming that the proportion of 0.995 to 1.0 is perfect [20]. Finally, we matched 7,540 dipyridamole users and 15,080 dipyridamole nonusers (Figure 1).

\section{Protective effects of dipyridamole in patients with advanced CKD}

During the study period, the total follow-up summation was 30,143 person-years. The mean followup time was 13.9 months in the dipyridamole users and 12.5 months in the nonusers. A total of 20,152 (70.7\%) patients progressed to ESRD, necessitating long-term dialysis, and 5,697 (20.0\%) died before a progression to ESRD required long-term dialysis (Table 2). The incidence of long-term dialysis was 69.7 per 100 person-years in the dipyridamole users and 72.5 per 100 person-years in the nonusers. The Kaplan-Meier survival curve revealed that patients treated with dipyridamole exhibited a significantly decreased risk of requiring chronic dialysis (Figure 2A). Compared with the nonusers, the dipyridamole users exhibited a lower chance of progression to ESRD requiring maintenance dialysis (adjusted HR, $0.97 ; 95 \%$ CI $0.94-1.00$ ), and the results remained consistent after propensity score-matching (adjusted HR, 0.96 ; 95\% CI, 0.93-0.99) (Table 2). On the dose-response relationship, we found that respective HRs of long-term dialysis related to dipyridamole use were significantly lower in cumulative defined daily doses (DDDs) $\geq 140$ within 90 days (adjusted HR, 0.91; 95\% CI, 0.87-0.95) or a prescribed daily dose of $\geq 75 \mathrm{mg}$ (adjusted HR, 0.91; 95\% CI, 0.88-0.95) compared to the dipyridamole nonusers (Table 2).

Table 2 shows the association between dipyridamole administration and pre-dialysis death. The incidence of death was 19.4 per 100 person-years in the dipyridamole 
users and 21.4 per 100 person-years in the nonusers. The Kaplan-Meier survival curve disclosed that the dipyridamole users had a significantly lower chance of death (Figure 2B). Compared with the nonusers, the dipyridamole users exhibited a decreased risk of death (adjusted HR, 0.90; 95\% CI 0.85-0.95), and the results remained consistent after propensity score-matching (adjusted HR, 0.91; 95\% CI, 0.85-0.97) (Table 2). On the dose-response relationship, we found that the respective HRs of pre-dialysis death related to dipyridamole use were significantly lower in cumulative DDDs $\geq 140$ within 90 days (adjusted HR, 0.86; 95\% CI, 0.80-0.94) or a prescribed daily dose of $\geq 75 \mathrm{mg}$ (adjusted HR, 0.87; 95\% CI, 0.81-0.95) compared to the dipyridamole nonusers (Table 2). In the stratified analyses, the decreased HRs of chronic dialysis and death in the dipyridamole cohort were generally consistent across nearly all subgroups (Figure 3). Finally, in the sensitivity analyses, the results were consistent with the main findings. The estimated effects of dipyridamole use on the primary outcomes were similar regardless of different exposure time for dipyridamole use calculated (Supplementary Tables 2-4).

\section{Interaction of dipyridamole and RAAS blockade on the risk of long-term dialysis and death in CKD 5 ND patients}

In the assessment of the interaction between dipyridamole and ACEI/ARB, we found that either ACEI/ ARB or dipyridamole administration was significantly associated with a lower risk for chronic dialysis compared to the patients who had taken neither ACEI/ARB nor dipyridamole. Moreover, concurrent use of an ACEI/ $\mathrm{ARB}$ and dipyridamole significantly diminished the risk of progression to ESRD (Figure 4A). In addition, either dipyridamole monotherapy or the concurrent use of ACEI/ ARB was significantly associated with a lower risk of death compared to those not taking ACEI/ARB and dipyridamole. However, ACEI/ARB use alone was not significantly associated with a lower risk of death (Figure 4B).

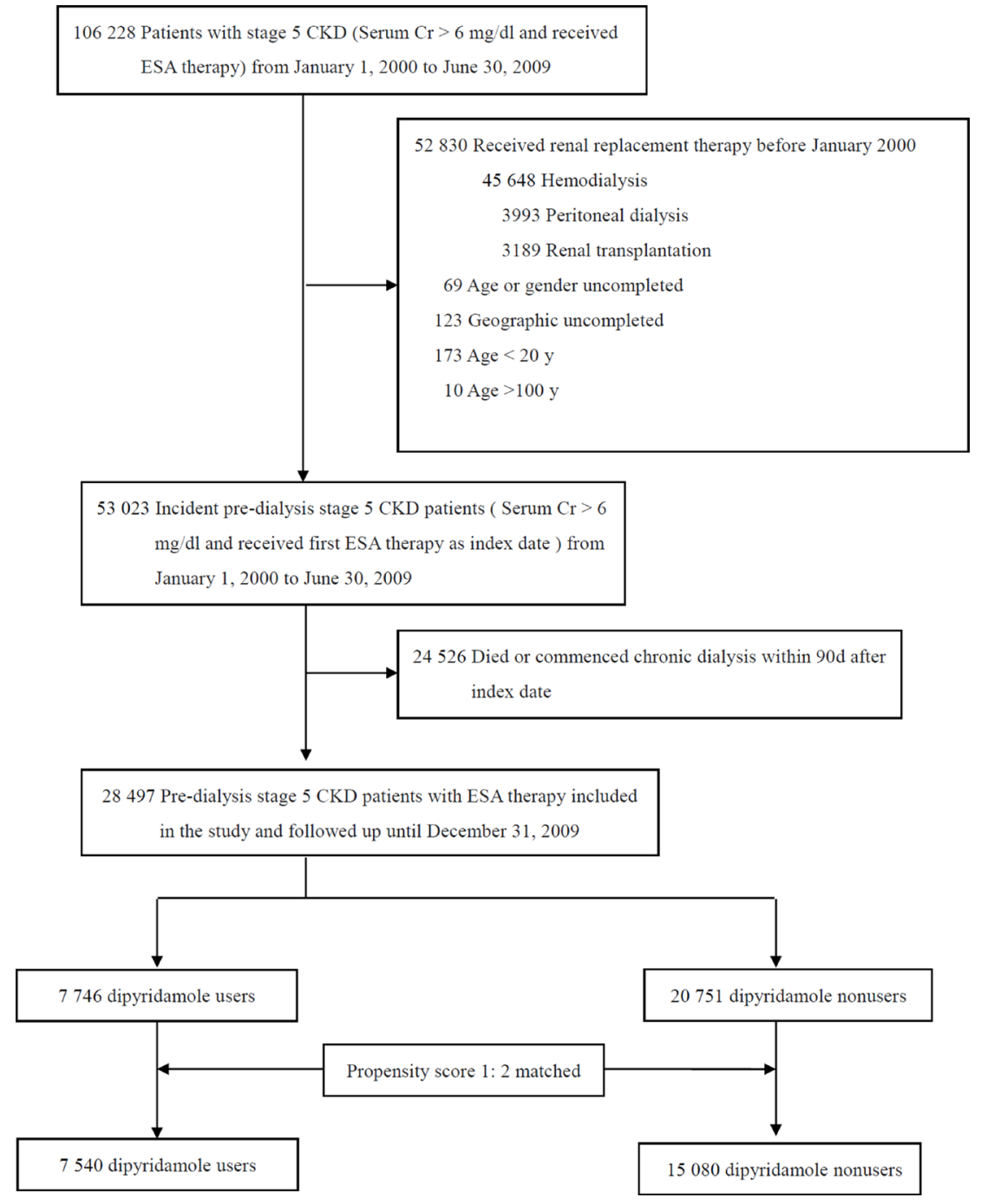

Figure 1: Study profile. Abbreviations: CKD, chronic kidney disease; Cr, creatinine; ESA, erythropoiesis stimulating agent. 
Table 1: Baseline characteristics of study subjects before and after propensity score matching by dipyridamole use

\begin{tabular}{|c|c|c|c|c|c|c|c|c|}
\hline & \multicolumn{4}{|c|}{ Before matching } & \multicolumn{4}{|c|}{ Propensity score-matched } \\
\hline & $\begin{array}{c}\text { Dipyridamole users } \\
(n=7,746)\end{array}$ & $\begin{array}{l}\text { Dipyridamole nonusers } \\
\qquad(n=\mathbf{2 0 , 7 5 1})\end{array}$ & $P$ & $S D$ & $\begin{array}{c}\text { Dipyridamole users } \\
(n=7,540)\end{array}$ & $\begin{array}{l}\text { Dipyridamole nonusers } \\
\qquad(n=15,080)\end{array}$ & $P$ & $S D$ \\
\hline Age, mean (SD), y & $66(12.7)$ & $65.2(13.2)$ & $<0.001$ & 0.059 & $65.9(12.8)$ & $65.9(13.1)$ & 0.82 & 0.003 \\
\hline \multicolumn{9}{|l|}{ Age group, y } \\
\hline $20-44$ & $431(5.6)$ & $1,445(7.0)$ & $<0.001$ & 0.058 & $430(5.7)$ & $975(6.5)$ & 0.03 & 0.032 \\
\hline $45-64$ & $2,773(35.8)$ & $7,793(37.6)$ & 0.006 & 0.036 & $2,712(36.0)$ & $5,361(35.6)$ & 0.54 & 0.009 \\
\hline $65-74$ & $2,385(30.8)$ & $5,933(28.6)$ & 0.001 & 0.048 & $2,317(30.7)$ & $4,427(29.4)$ & 0.03 & 0.030 \\
\hline $75-100$ & $2,157(27.9)$ & $5,580(26.9)$ & 0.11 & 0.022 & $2,081(27.6)$ & $4,317(28.6)$ & 0.11 & 0.023 \\
\hline \multicolumn{9}{|l|}{ Gender } \\
\hline Male, $n(\%)$ & $3,645(47.1)$ & $9,647(46.5)$ & 0.39 & 0.012 & $3,529(46.8)$ & $7,055(46.8)$ & 0.98 & 0.000 \\
\hline \multicolumn{9}{|c|}{ Comorbid conditions within the 3 y preceding the index date } \\
\hline Diabetes, $n(\%)$ & $3,921(50.6)$ & $11,171(53.8)$ & $<0.001$ & 0.064 & $3,856(51.1)$ & $7,672(50.9)$ & 0.71 & 0.005 \\
\hline MI, $n(\%)$ & $2,134(27.6)$ & $5,110(24.6)$ & $<0.001$ & 0.067 & $2,027(26.9)$ & $4,030(26.7)$ & 0.80 & 0.004 \\
\hline Stroke, $n(\%)$ & $1,420(18.3)$ & $3,745(18.0)$ & 0.57 & 0.007 & $1,387(18.4)$ & $2,780(18.4)$ & 0.94 & 0.001 \\
\hline Cancer, $n(\%)$ & $646(8.3)$ & $2,011(9.7)$ & $<0.001$ & 0.047 & $644(8.5)$ & $1,244(8.2)$ & 0.45 & 0.011 \\
\hline \multicolumn{9}{|c|}{ Charlson Comorbidity Index score } \\
\hline$\leq 3, n(\%)$ & $2,903(37.5)$ & $7,929(38.2)$ & 0.26 & 0.015 & $2,853(37.8)$ & $5,754(38.2)$ & 0.64 & 0.007 \\
\hline $4-5, n(\%)$ & $1,392(18.0)$ & $3,814(18.4)$ & 0.43 & 0.011 & $1,363(18.1)$ & $2,665(17.7)$ & 0.45 & 0.011 \\
\hline$>5, n(\%)$ & $3,451(44.6)$ & $9,008(43.4)$ & 0.08 & 0.023 & $3,324(44.1)$ & $6,661(44.2)$ & 0.90 & 0.002 \\
\hline Mean (SD) & $4.4(2.3)$ & $4.3(2.3)$ & 0.001 & 0.043 & $4.4(2.2)$ & $4.4(2.3)$ & 0.89 & 0.002 \\
\hline \multicolumn{9}{|c|}{ Nephrologist visits within the 3 y preceding the index date } \\
\hline $0, n(\%)$ & $1,629(21.0)$ & $4,090(19.7)$ & 0.013 & 0.033 & $1,558(20.7)$ & $3,129(20.7)$ & 0.88 & 0.002 \\
\hline $1-6, n(\%)$ & $2,056(26.5)$ & $5,562(26.8)$ & 0.66 & 0.006 & $2,007(26.6)$ & $3,983(26.4)$ & 0.74 & 0.005 \\
\hline$>6, n(\%)$ & $4,061(52.4)$ & $11,098(53.5)$ & 0.11 & 0.021 & $3,975(52.7)$ & $7,968(52.8)$ & 0.87 & 0.002 \\
\hline \multicolumn{9}{|c|}{ Anti-hypertensive agents used } \\
\hline ACEI, $n(\%)$ & $1,873(24.2)$ & $4,041(19.5)$ & $<0.001$ & 0.114 & $1,696(22.5)$ & $3,450(22.9)$ & 0.52 & 0.009 \\
\hline $\mathrm{ARB}, n(\%)$ & $2,634(34.0)$ & $7,673(37.0)$ & $<0.001$ & 0.062 & $2,611(34.6)$ & $5,157(34.2)$ & 0.52 & 0.009 \\
\hline Beta-blockers, $n(\%)$ & $3,192(41.2)$ & $8,898(42.9)$ & 0.01 & 0.034 & $3,123(41.4)$ & $6,236(41.4)$ & 0.92 & 0.001 \\
\hline $\begin{array}{l}\text { Calcium channel } \\
\text { blockers, } n(\%)\end{array}$ & $5,915(76.4)$ & $15,955(76.9)$ & 0.35 & 0.012 & $5,768(76.5)$ & $11,515(76.4)$ & 0.82 & 0.003 \\
\hline Diuretics, $n(\%)$ & $4,817(62.2)$ & $13,446(64.8)$ & $<0.001$ & 0.054 & $4,741(62.9)$ & $9,423(62.5)$ & 0.57 & 0.008 \\
\hline Pentoxifylline, $n(\%)$ & $1,036(13.4)$ & $3,398(16.4)$ & $<0.001$ & 0.084 & $1,034(13.7)$ & $2,009(13.3)$ & 0.42 & 0.011 \\
\hline Insulin, $n(\%)$ & $1,679(21.7)$ & $5,225(25.2)$ & $<0.001$ & 0.083 & $1,671(22.0)$ & $3,321(22.0)$ & 0.81 & 0.003 \\
\hline Statin, $n(\%)$ & $1,275(16.5)$ & $3,681(17.7)$ & 0.01 & 0.034 & $1,263(16.8)$ & $2,492(16.4)$ & 0.67 & 0.006 \\
\hline Aspirin, $n(\%)$ & $1,556(20.1)$ & $4,194(20.2)$ & 0.82 & 0.003 & $1,520(20.2)$ & $3,057(20.3)$ & 0.84 & 0.003 \\
\hline Acetaminophen, $n(\%)$ & $3,960(51.1)$ & $10,704(51.6)$ & 0.49 & 0.009 & $3,861(51.1)$ & $7,753(51.3)$ & 0.92 & 0.001 \\
\hline \multicolumn{9}{|l|}{ NSAIDs, $n(\%)$} \\
\hline COX-2 inhibitors & $387(5.0)$ & $932(4.5)$ & 0.07 & 0.024 & $375(5.0)$ & $765(5.1)$ & 0.75 & 0.005 \\
\hline $\begin{array}{l}\text { Non-COX-2 } \\
\text { inhibitors }\end{array}$ & $2,882(37.2)$ & $7,198(34.7)$ & $<0.001$ & 0.053 & $2,751(36.5)$ & $5,543(36.8)$ & 0.69 & 0.006 \\
\hline \multicolumn{9}{|l|}{ Geographic location } \\
\hline Northern, $n(\%)$ & $3,251(42.0)$ & $9,017(43.5)$ & 0.025 & 0.030 & 3,235 (42.9) & $6,466(42.9)$ & 0.97 & 0.001 \\
\hline Middle, $n(\%)$ & $2,034(26.3)$ & $4,183(20.2)$ & $<0.001$ & 0.145 & $1,847(24.5)$ & $3.711(24.6)$ & 0.85 & 0.003 \\
\hline Southern, $n(\%)$ & $2,349(30.3)$ & $7,110(34.3)$ & $<0.001$ & 0.084 & $2,347(30.9)$ & $4.685(31.1)$ & 0.93 & 0.001 \\
\hline $\begin{array}{l}\text { Eastern or other } \\
\text { islands, } n(\%)\end{array}$ & $111(1.4)$ & $442(2.1)$ & 0.001 & 0.053 & $111(1.5)$ & $218(1.4)$ & 0.88 & 0.002 \\
\hline Propensity score & $0.733(0.056)$ & $0.716(0.058)$ & $<0.001$ & 0.285 & $0.721(0.053)$ & $0.719(0.055)$ & 0.13 & 0.021 \\
\hline
\end{tabular}

$\mathrm{ACEI}=$ angiotensin converting enzyme inhibitor. $\mathrm{ARB}=$ angiotensin $\mathrm{II}$ receptor blocker. $\mathrm{CCI}=\mathrm{Charlson}$ comorbidity index. $\mathrm{CKD}=$ chronic kidney disease. $\mathrm{COX}-2=$ cyclooxygenase $-2 . \mathrm{MI}=$ myocardial infarction. NSAID $=$ non-steroidal anti-inflammatory drug. $\mathrm{SD}=$ standardized difference. 


\section{Risk of bleeding in CKD 5 ND patients receiving dipyridamole}

During the median follow-up period of 17.8 months, a total of 3,615 (12.7\%) patients had bleeding events (Supplementary Table 5). Compared with the dipyridamole nonusers, the risk of bleeding in dipyridamole users did not significantly increase (adjusted HR, 0.99; 95\% CI $0.92-1.06$ ). After the propensity score-matching, the dipyridamole users still did not have a significantly higher chance for bleeding (adjusted HR, 1.00; 95\% CI, 0.92-1.08).

\section{DISCUSSION}

The mechanism of the reno-protective effects of dipyridamole has been explained by several animal and human studies. Dipyridamole increased the local concentration of adenosine, which stimulated adenylyl cyclase in platelets, leading to increased intracellular levels of cyclic adenosine monophosphate (cAMP) [21]. By inhibiting phosphodiesterase (PDE), dipyridamole increases prostacyclin $\left(\mathrm{PGI}_{2}\right)$ production and cyclic guanine monophosphate (cGMP) levels on vascular smooth muscle, leading to vasodilation [22, 23]. Moreover, by increasing intracellular levels of cGMP, dipyridamole can augment many of the downstream signaling pathways of nitric oxide [14]. Hewitson et al. disclosed that dipyridamole inhibits profibrotic activities of renal fibroblasts and collagen synthesis in vitro [24]. In animal models, dipyridamole monotherapy or combinations with ACEI therapy attenuated microalbuminuria and enhanced eNOS expression in streptozotocin-induced diabetic rats [25]. In rats with subtotal nephrectomy, dipyridamole or ACEI therapy markedly improved renal function. Further histological examination of the remnant kidney detected the presence of vasodilation with a lower degree of podocyte swelling in both dipyridamole and ACEI treatment groups. These data indicated that dipyridamole still attenuated the progression of glomerular disease in advanced CKD in rats [26]. In humans, dipyridamole monotherapy reduced urinary albumin excretion in diabetes patients with normo- or microalbuminuria [17]. In two meta-analyses of antiplatelet therapy for IgA nephropathy, dipyridamole therapy was beneficial for reducing the risk of proteinuria $[27,28]$. In addition, dipyridamole combination therapy with ACE-I, antiplatelet agents or immunosuppressants significantly reduced proteinuria in patients with IgA nephropathy

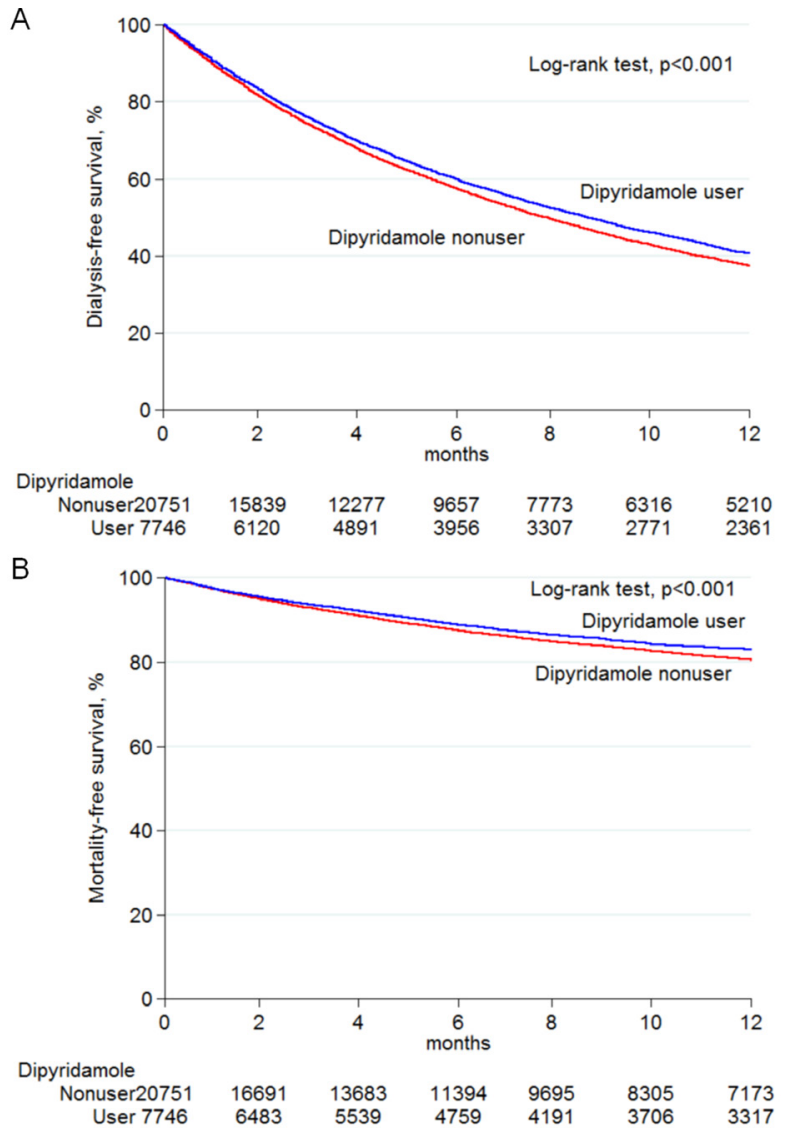

Figure 2: Kaplan-Meier analysis of survival curves among pre-dialysis stage 5 CKD patients. Dialysis-free (A) and mortality-free (B) survivals constitute the study end points. Difference between dipyridamole users and non-users was analyzed by log-rank test. Abbreviations: CKD, chronic kidney disease. 
Table 2: Risk of long-term dialysis and death among patients with advanced CKD comparing dipyridamole users vs. nonusers

\begin{tabular}{|c|c|c|c|c|c|c|c|}
\hline & \multicolumn{4}{|c|}{ Before Matching } & \multicolumn{3}{|c|}{ After Matching } \\
\hline & $N$ of event & $\begin{array}{l}\text { Incidence rate } \\
\text { (100 person- } \\
\text { years) }\end{array}$ & $\begin{array}{c}\text { Crude HR } \\
(95 \% \text { CI) }\end{array}$ & $\begin{array}{c}\text { Adjusted HR } \\
(95 \% \text { CI) }\end{array}$ & $N$ of event & $\begin{array}{l}\text { Incidence rate } \\
\text { (100 person- } \\
\text { years) }\end{array}$ & $\begin{array}{c}\text { Adjusted HR } \\
\text { (95\% CI) }\end{array}$ \\
\hline \multicolumn{8}{|l|}{ Long-term dialysis } \\
\hline Dipyridamole nonuser & 14,463 & 74.26 & 1.0 (reference) & 1.0 (reference) & 10,480 & 72.48 & 1.0 (reference) \\
\hline Dipyridamole user & 5,689 & 69.35 & $0.94(0.91-0.97)^{*}$ & $0.97(0.94-1.00)^{*}$ & 5,531 & 69.73 & $0.96(0.93-0.99)^{*}$ \\
\hline$<140 \mathrm{DDD}$ & 2,768 & 72.49 & $0.98(0.94-1.02)$ & $1.02(0.98-1.06)$ & 2,695 & 73.18 & $1.02(0.98-1.06)$ \\
\hline$\geq 140 \mathrm{DDD}$ & 2,921 & 66.61 & $0.91(0.87-0.95)^{*}$ & $0.92(0.88-0.96)^{*}$ & 2,836 & 66.74 & $0.91(0.87-0.95)^{*}$ \\
\hline$<75 \mathrm{mg} /$ day & 2,617 & 72.90 & $0.99(0.95-1.03)$ & $1.02(0.98-1.07)$ & 2,546 & 73.66 & $1.03(0.98-1.07)$ \\
\hline$\geq 75 \mathrm{mg} /$ day & 3,072 & 66.58 & $0.91(0.87-0.94)^{*}$ & $0.92(0.89-0.96)^{*}$ & 2,985 & 66.70 & $0.91(0.88-0.95)^{*}$ \\
\hline \multicolumn{8}{|l|}{ Death } \\
\hline Dipyridamole nonuser & 4,116 & 21.13 & 1.0 (reference) & 1.0 (reference) & 3,087 & 21.35 & 1.0 (reference) \\
\hline Dipyridamole user & 1,581 & 19.27 & $0.91(0.86-0.96)^{*}$ & $0.90(0.85-0.95)^{*}$ & 1,536 & 19.37 & $0.91(0.85-0.97)^{*}$ \\
\hline$<140 \mathrm{DDD}$ & 828 & 21.69 & $1.02(0.95-1.10)$ & $0.95(0.88-1.02)$ & 800 & 21.72 & $0.95(0.88-1.03)$ \\
\hline$\geq 140 \mathrm{DDD}$ & 753 & 17.17 & $0.81(0.75-0.87)^{*}$ & $0.85(0.79-0.92)^{*}$ & 736 & 17.32 & $0.86(0.80-0.94)^{*}$ \\
\hline$<75 \mathrm{mg} /$ day & 781 & 21.76 & $1.03(0.95-1.11)$ & $0.94(0.87-1.02)$ & 754 & 21.81 & $0.95(0.87-1.03)$ \\
\hline$\geq 75 \mathrm{mg} /$ day & 800 & 17.34 & $0.82(0.76-0.88)^{*}$ & $0.86(0.80-0.93)^{*}$ & 782 & 17.47 & $0.87(0.81-0.95)^{*}$ \\
\hline
\end{tabular}

$\mathrm{CI}=$ confidence interval. $\mathrm{CKD}=$ chronic kidney disease. $\mathrm{DDD}=$ defined daily doses. $\mathrm{HR}=$ hazard ratio

A multivariate analysis was adjusted for all variables listed in Table 1.

${ }^{*} P<0.05$ compared with dipyridamole nonusers

and primary membranoproliferative glomerulonephritis $[18,19,29,30]$. However, our knowledge about the reno-protective effect of dipyridamole use is limited in pre-dialysis, advanced CKD patients.

Limited studies have disclosed the long-term outcome of dipyridamole use other than its effects on decreasing proteinuria or hastening the GFR decline rate in CKD patients who had not yet received dialysis. This is not surprising, because the previous studies were small in scale, and all the study periods were short. To our knowledge, our national cohort study first demonstrated that dipyridamole treatment was associated with a $4 \%$ lower risk of long-term dialysis and a $9 \%$ lower risk of death in CKD 5 ND patients. Moreover, the risk of bleeding events was not significantly increased in dipyridamole users. A small-scale observational study demonstrated that the use of dipyridamole provided a better renal outcome in CKD patients with a mean eGFR of $25.5 \mathrm{ml} /$ minute $/ 1.73 \mathrm{~m}^{2}$ [31]. The authors also showed that dipyridamole users exhibited a decreased
A

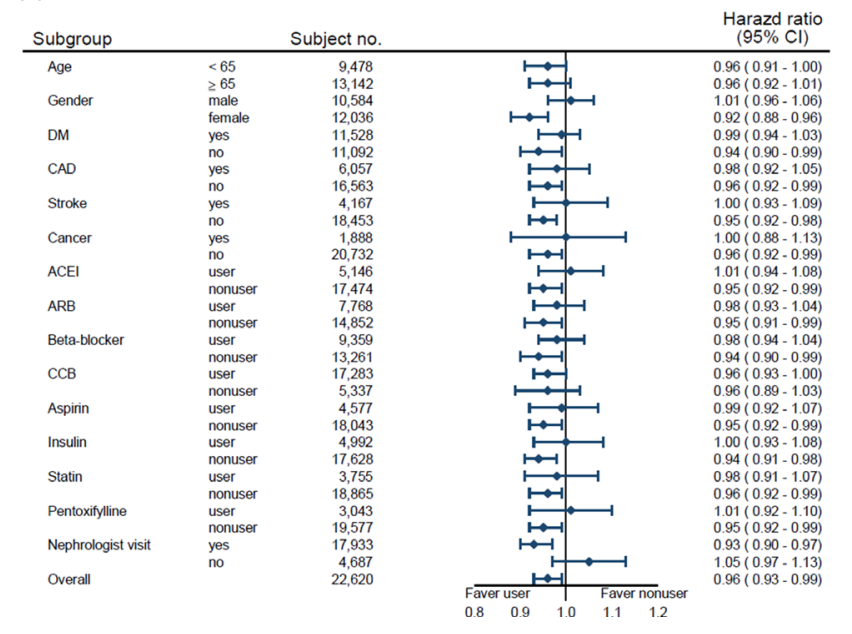

B

\begin{tabular}{|c|c|c|c|c|c|}
\hline \multirow{2}{*}{$\frac{\text { Subgroup }}{\text { Age }}$} & \multicolumn{2}{|c|}{ Subject no. } & \multicolumn{3}{|r|}{$\begin{array}{c}\text { Harazd ratio } \\
(95 \% \mathrm{Cl})\end{array}$} \\
\hline & $<65$ & 9,478 & $\longmapsto$ & & $0.89(0.79-1.01)$ \\
\hline & $\geq 65$ & 13,142 & & & $0.92(0.86-0.99)$ \\
\hline Gender & male & 10,584 & $\longmapsto$ & & $0.84(0.77-0.92)$ \\
\hline DM & $\begin{array}{l}\text { female } \\
\text { yes }\end{array}$ & $\begin{array}{l}12,036 \\
11,528\end{array}$ & & & $\begin{array}{l}0.97(0.89-1.06) \\
090(082-0.98)\end{array}$ \\
\hline & $\begin{array}{l}\text { yes } \\
\text { no }\end{array}$ & $\begin{array}{l}11,092 \\
11,092\end{array}$ & & & $0.93(0.85-1.02)$ \\
\hline CAD & yes & 6,057 & & H & $0.92(0.83-1.03)$ \\
\hline & & 16,563 & $\longmapsto$ & & $0.90(0.84-0.97)$ \\
\hline Stroke & yes & 4,167 & & $\longrightarrow$ & $0.95(0.84-1.08)$ \\
\hline & no & 18,453 & $\longmapsto$ & & $0.90(0.84-0.96)$ \\
\hline Cancer & yes & 1,888 & & -1 & $0.87(0.73-1.04)$ \\
\hline & & 20,732 & $\longmapsto 1$ & & $0.92(0.86-0.98)$ \\
\hline ACEI & $\begin{array}{l}\text { user } \\
\text { nonuser }\end{array}$ & $\begin{array}{r}5,146 \\
17,474\end{array}$ & $\mapsto$ & & $\begin{array}{l}0.90(0.80-1.02) \\
090(084-0.97\end{array}$ \\
\hline ARB & 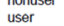 & $\begin{array}{r}71,474 \\
7,768\end{array}$ & & & $0.88(0.79-0.99)$ \\
\hline & nonuser & 14,852 & $\longmapsto$ & & $0.93(0.86-1.00)$ \\
\hline Beta-blocker & user & 9,359 & & & $1.02(0.92-1.12)$ \\
\hline & nonuser & 13,261 & $\longmapsto$ & & $0.84(0.78-0.91)$ \\
\hline CCB & user & $\begin{array}{l}17,283 \\
5.207\end{array}$ & $\longmapsto$ & & $0.92(0.86-0.99)$ \\
\hline & nonuser & 5,337 & + & & $0.87(0.78-0.98)$ \\
\hline Aspirin & $\begin{array}{l}\text { user } \\
\text { nonuser }\end{array}$ & $\begin{array}{r}4,577 \\
18043\end{array}$ & $\longmapsto$ & $\longrightarrow$ & $\begin{array}{l}0.93(0.82-1.06) \\
0.91(085-0.98\end{array}$ \\
\hline Insulin & & $\begin{array}{r}18,043 \\
4,992\end{array}$ & 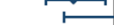 & & $1.01(0.89-1.13)$ \\
\hline & nonuser & 17,628 & $\longmapsto$ & & $0.89(0.82-0.95)$ \\
\hline Statin & & 3,755 & & $\longrightarrow$ & $0.93(0.78-1.11)$ \\
\hline & nonuser & 18,865 & $\longmapsto$ & & $0.91(0.85-0.97)$ \\
\hline Pentoxifylline & user & 3,043 & & & $1.05(0.88-1.25)$ \\
\hline & nonuser & 19,577 & $\longmapsto$ & & $0.89(0.83-0.95)$ \\
\hline Nephrologist visit & yes & 17,933 & $\longmapsto$ & & $0.93(0.87-1.00)$ \\
\hline & no & 4,687 & $\longmapsto$ & & $0.87(0.78-0.98)$ \\
\hline Overall & & 22,620 & 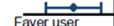 & & $0.91(0.85-0.97)$ \\
\hline & & & $\begin{array}{l}\text { Faver user } \\
0.8 \quad 0.9\end{array}$ & $\begin{array}{l}\text { Fave } \\
111\end{array}$ & \\
\hline
\end{tabular}

Figure 3: Adjusted hazard ratios of long-term dialysis (A) and death (B) among pre-dialysis stage 5 CKD patients by dipyridamole use. Abbreviations; ACEI, angiotensin converting enzyme inhibitor; ARB, angiotensin II receptor blocker; CAD, coronary artery disease; CCB, calcium channel blocker; CI, confidence interval; CKD, chronic kidney disease; DM, diabetes mellitus; HR, hazard ratios. 
risk of death compared to non-users. Whether their result could be extrapolated to stage $5 \mathrm{ND}$ was questionable. In contrast, our study not only extends the current knowledge in the field but also demonstrates the consistency and generalizability of the effectiveness of dipyridamole in patients with early stage CKD to CKD 5 ND.

From a clinical viewpoint, several issues merit discussion in this study. First, for patients with rapidly declining renal function and low GFR, such as CKD 5 ND patients, physicians usually do not prescribe RAAS blockade, and these patients were excluded in many studies. This is the reason why research about medications in pre-dialysis advanced CKD patients is limited. However, our previous study demonstrated for the first time that ACEI/ARB users exhibited a $6 \%$ lower risk of long-term dialysis or death. In the present study, we demonstrated that dipyridamole represents a promising and safe agent for reno-protection, either as monotherapy or in combination with RAAS blockade. Second, some CKD patients cannot tolerate ACEI/ARB use because of hypotension, hyperkalemia or renal artery stenosis. Dipyridamole treatment might be a better choice for reno-protection in these situations. Third, we found that dipyridamole monotherapy, but not RAAS blockade, was associated with low death risk in CKD 5 ND patients. The possible causes might be related to the stimulation of nitric oxide action and the inhibition of platelet aggregation via the inhibition of phosphodiesterase and the antioxidant effect of dipyridamole [14-16]. Our results indicated that these platelet and non-platelet actions of dipyridamole may contribute to its translational therapeutic benefits not only in patients with traditional vascular disease but also in patients with advanced CKD.

Some limitations should be addressed. First, since our cohort study is observational, it cannot prove causality. However, to conduct a randomized controlled trial with adequate statistical power $(\alpha=0.05 ; 1-\beta=0.8)$, we needed recruiting at least $21,806 \mathrm{CKD} 5 \mathrm{ND}$ patients to examine a $9 \%$ relative risk reduction of death. It seems impracticable to conduct such a large-scale trial in this population. Moreover, some researches reveal that well-designed

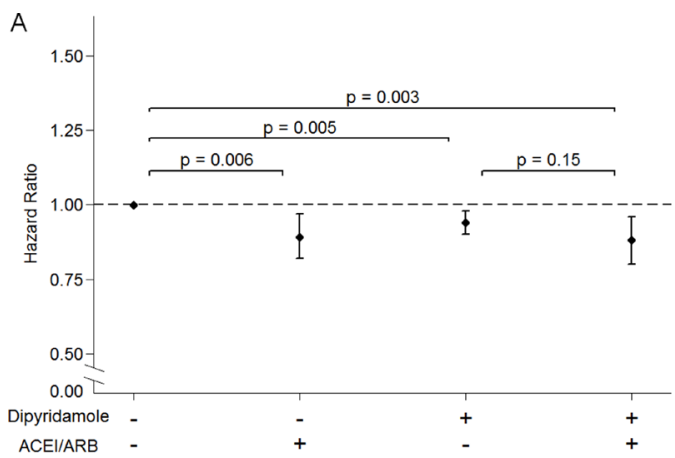

observational cohort studies may generate comparable outcomes [32, 33]. Second, although some risk factors for predicting CKD progression, such as proteinuria or renal function, are not available in the present study. We did a propensity score-based matched design to minimize these confounders. In addition, the findings of sensitivity and subgroup analyses generally support the robustness of our results; however, neutral effects of dipyridamole use could be seen in some subgroup analyses, indicating we rather be more conservative to make our conclusion. Randomized clinical trials are needed to validate our findings in the future. Moreover, nephrologists usually prescribe dipyridamole in patients with proteinuria, the use of dipyridamole in patients with more proteinuria will bias the result toward the null hypothesis. Third, some patients with transient creatinine concentrations of $>6$ $\mathrm{mg} / \mathrm{dL}$ might be recruited in this cohort. Therefore, we had restricted our analysis to subjects who received ESA at least two consecutive visits, and the result remained unchanged. Finally, the generalizability of our study is limited to advanced CKD patients who are anemic. Our results can't be applicable to all stage 5 CKD patients who had not yet received dialysis.

In conclusion, our nationwide cohort study extend the knowledge of dipyridamole therapy from early-stage CKD patients to CKD 5 ND patients. Our study reveals that either dipyridamole monotherapy or the combination use of dipyridamole and RAAS blockade were significantly associated with a decreased risk of long-term dialysis and pre-dialysis death in CKD 5 ND patients. Moreover, the risk of bleeding events was not significantly increased in dipyridamole users. Further randomized controlled studies may be needed to provide definitive results.

\section{MATERIALS AND METHODS}

\section{Data source}

Patient's data were retrieved from Taiwan NHI Research Database, which contains the health-care data

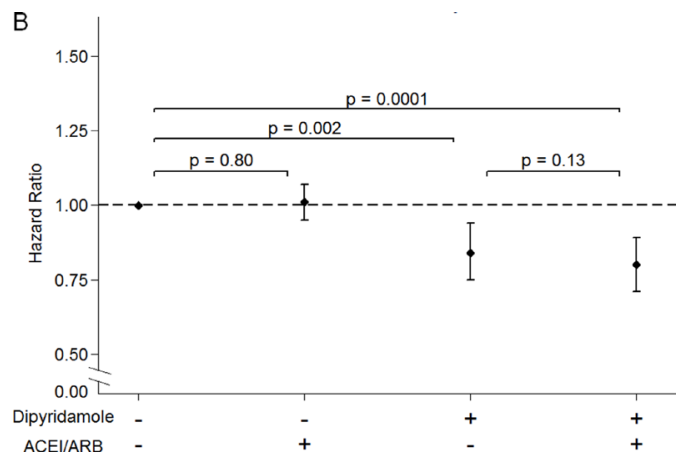

Figure 4: The interaction of dipyridamole and the renin-angiotensin-aldosterone system blockade on the risk of long-term dialysis (A) and death (B) among pre-dialysis stage 5 CKD patients. Abbreviations; ACEI, angiotensin converting enzyme inhibitor; ARB, angiotensin II receptor blocker; HR, hazard ratios. 
gathered prospectively for $99 \%$ of the entire population of 23 million people [34]. De-identified information recorded in the NHI Research Database includes diagnostic codes according to the International Classification of Diseases9th revision (ICD-9), birthday, gender, residency area, drug prescriptions, and medical procedures. The study methods were carried out in accordance with the approved guidelines for research involving human subjects from the Taiwan Ministry of Health and Welfare. The Institutional Review Board at Taipei Tzu Chi Hospital approved the study. Informed consent was waived due to the deidentification of any personal information in this database.

\section{Design and study participants}

We selected patients who had CKD and received ESA therapy in the NHI Research Database between January 1, 2000 and June 30, 2009, and then were followed up until December 31, 2009. Taiwan NHI reimbursement regulations state that ESA treatment can be initiated when patients with CKD who do not need dialysis have a serum creatinine concentration of $>6 \mathrm{mg} / \mathrm{dL}$ (approximately equivalent to an estimated GFR of $<15 \mathrm{ml} / \mathrm{min}$ per 1.73 $\mathrm{m}^{2}$ ) and a hematocrit of $<28 \%$, to maintain a hematocrit not exceeding $36 \%$. The selected cohort had been described in our previous study $[13,35]$. According to the record by the Taiwan Ministry of Health and Welfare, $85 \%$ of CKD 5 ND patients received a prescription for ESA. Therefore, this indicated that our selected cohort was highly representative of patients with stage 5 CKD in Taiwan [36]. We defined the first day of ESA administration as the index date. Our study excluded individuals younger than 20 years or older than 100 years, those who received dialysis or kidney transplantation before treatment with ESA, and individuals who died or who begun renal replacement therapy within 90 days of first dose of ESA prescription. We defined comorbid disorders, including diabetes, myocardial infarction, stroke, and cancer, which had been diagnosed within 3 years preceding the index date. We used Charlson comorbidity index (CCI) to quantify patients' comorbidity profiles [37].

\section{Exposure assessment}

Patients who had taken dipyridamole within 90 days of the index date were defined as dipyridamole users ( $n=7,746)$, and the remaining subjects were defined as dipyridamole nonusers $(n=20,751)$. Furthermore, to assess dose-effect, we analyzed the risk of chronic dialysis and death according to the cumulative DDD during the 90-day exposure period ( $<140 \mathrm{DDD}, \geq 140 \mathrm{DDD})$ and the prescribed daily dose $(<75 \mathrm{mg}, \geq 75 \mathrm{mg})$, relative to no dipyridamole use. DDD, a technical unit of measurement, is defined as the assumed average maintenance dose per day for a drug used for its main indication in adults, as previously described [35].

\section{Renal outcome and mortality}

The observation period begun 90 days after the index date until the initiation of maintenance dialysis, death, or December 31, 2009, whichever happened first. The primary outcomes were long-term dialysis or predialysis death. The onset of renal outcome was defined as the date of initiation of long-term dialysis for at least 90 days. The onset of mortality was defined as the date of death.

\section{Statistical analysis}

The baseline characteristics were compared with the 2-sided $t$ test and the chi-square test. We defined the study entry as the 90th day after the index date. Patient follow-up visits happened until the time of long-term dialysis, death or December 31, 2009. The primary outcomes were long-term chronic dialysis and pre-dialysis death. We used Cox's proportional hazard models to compare renal outcome and death while controlling for baseline covariates. We expressed results as HRs with 95\% CIs, compared with dipyridamole nonusers. We evaluated proportional hazard assumption by comparing estimated log-log survival curves for all time-independent covariates. Because we expected dipyridamole users and nonusers to differ with respect to prognostic factors confound the outcome analyses, we used a propensity score-based matching to control residual confounding factors. We incorporated all baseline characteristics listed in Table 1 into our analysis as independent variables. We deemed a two-sided $P$ values less than 0.05 significant. We performed statistical analyses with SAS version 9.3, and STATA SE version 14.

\section{Subgroup and sensitivity analyses}

To evaluate effect modification, we did subgroup analyses in pre-specified strata of clinical interest, including age, gender, the presence or absence of diabetes mellitus, CAD, stroke, cancer, the use of anti-hypertensive medications, aspirin, insulin, statin and pentoxifylline use, and nephrologist care. To assess the reliability of our findings, we performed a series of analyses to define dipyridamole administration at intervals of 30, 60 days and 120 days after first ESA use to minimize misclassification bias (Supplementary Tables 2-4).

\section{Author contributions}

Wrote the manuscript: K.L.K.; designed the research; K.L.K., S.C.H., W.C.T., C.C.H., D.C.T.; performed the research; K.L.K., J.S.L., C.C.H., D.C.T.; analyzed the data: S.C.H. J.S.L., M.H.L.; contributed new reagents/analytical tools. C.C.H., D.C.T.; funding: K.L.K., D.C.T. 


\section{CONFLICTS OF INTEREST}

The authors declare no conflicts of interest.

\section{FINANCIAL SUPPORT}

This work was supported by grants from the Ministry of Science and Technology, R.O.C. (MOST 102-2314-B-010-004-MY3, MOST 105-2314-B-010-016 and MOST 105-2314-B-303-013-MY3), Taipei Veterans General Hospital (V104E4-001 and V105C-013), Taipei Tzu Chi General Hospital (TCRD-TPE-103-RT-4, and TCRD-TPE-105-19), and Foundation for Poison Control.

\section{REFERENCES}

1. Stenvinkel P. Chronic kidney disease: a public health priority and harbinger of premature cardiovascular disease. J Intern Med. 2010; 268:456-467.

2. Kagami S, Border WA, Miller DE, Noble NA. Angiotensin II stimulates extracellular matrix protein synthesis through induction of transforming growth factor-beta expression in rat glomerular mesangial cells. J Clin Invest. 1994; 93:2431-2437.

3. Anders HJ, Vielhauer V, Schlondorff D. Chemokines and chemokine receptors are involved in the resolution or progression of renal disease. Kidney Int. 2003; 63:401-415.

4. Zeisberg M, Hanai J, Sugimoto H, Mammoto T, Charytan D, Strutz F, Kalluri R. BMP-7 counteracts TGF-beta1-induced epithelial-to- mesenchymal transition and reverses chronic renal injury. Nat Med. 2003; 9:964-968.

5. Ostendorf T, Rong S, Boor P, Wiedemann S, Kunter U, Haubold U, van Roeyen CR, Eitner F, Kawachi H, Starling G, Alvarez E, Smithson G, Floege J. Antagonism of PDGF-D by human antibody CR002 prevents renal scarring in experimental glomerulonephritis. J Am Soc Nephrol. 2006; 17:1054-1062.

6. James PA, Oparil S, Carter BL, Cushman WC, DennisonHimmelfarb C, Handler J, Lackland DT, LeFevre ML, MacKenzie TD, Ogedegbe O, Smith SC Jr, Svetkey LP, Taler SJ, et al. 2014 evidence-based guideline for the management of high blood pressure in adults: report from the panel members appointed to the Eighth Joint National Committee (JNC 8). JAMA. 2014; 311:507-520.

7. Brenner BM, Cooper ME, de Zeeuw D, Keane WF, Mitch WE, Parving HH, Remuzzi G, Snapinn SM, Zhang Z, Shahinfar S; RENAAL Study Investigators. Effects of losartan on renal and cardiovascular outcomes in patients with type 2 diabetes and nephropathy. N Engl J Med. 2001; 345:861-869.

8. Lewis EJ, Hunsicker LG, Clarke WR, Berl T, Pohl MA, Lewis JB, Ritz E, Atkins RC, Rohde R, Raz I; Collaborative Study Group. Renoprotective effect of the angiotensinreceptor antagonist irbesartan in patients with nephropathy due to type 2 diabetes. N Engl J Med. 2001; 345:851-860.
9. Ruggenenti P, Perna A, Gherardi G, Garini G, Zoccali C, Salvadori M, Scolari F, Schena FP, Remuzzi G. Renoprotective properties of ACE-inhibition in non-diabetic nephropathies with non-nephrotic proteinuria. Lancet. 1999; 354:359-364.

10. Randomised placebo-controlled trial of effect of ramipril on decline in glomerular filtration rate and risk of terminal renal failure in proteinuric, non-diabetic nephropathy. The GISEN Group (Gruppo Italiano di Studi Epidemiologici in Nefrologia). Lancet. 1997; 349:1857-1863.

11. Hou FF, Zhang X, Zhang GH, Xie D, Chen PY, Zhang WR, Jiang JP, Liang M, Wang GB, Liu ZR, Geng RW. Efficacy and safety of benazepril for advanced chronic renal insufficiency. N Engl J Med. 2006; 354:131-140.

12. Hou FF, Xie D, Zhang X, Chen PY, Zhang WR, Liang M, Guo ZJ, Jiang JP. Renoprotection of optimal Antiproteinuria Doses (ROAD) study: a randomized controlled study of benazepril and losartan in chronic renal insufficiency. J Am Soc Nephrol. 2007; 18:1889-1898.

13. Hsu TW, Liu JS, Hung SC, Kuo KL, Chang YK, Chen YC, Hsu CC, Tarng DC. Renoprotective effect of reninangiotensin-aldosterone system blockade in patients with predialysis advanced chronic kidney disease, hypertension and anemia. JAMA Intern Med. 2014; 174:347-354.

14. Harker LA, Kadatz RA. Mechanism of action of dipyridamole. Thromb Res Suppl. 1983; 4:39-46.

15. Bult H, Fret HR, Jordaens FH, Herman AG. Dipyridamole potentiates the anti-aggregating and vasodilator activity of nitric oxide. Eur J Pharmacol. 1991; 199:1-8.

16. Ruggiero AC, Nepomuceno MF, Jacob RF, Dorta DJ, Tabak M. Antioxidant effect of dipyridamole (DIP) and its derivative RA 25 upon lipid peroxidation and hemolysis in red blood cells. Physiol Chem Phys Med NMR. 2000; 32:35-48.

17. Aizawa T, Suzuki S, Asawa T, Komatsu M, Shigematsu S, Okada N, Katakura M, Hiramatsu K, Shinoda T, Hashizume $\mathrm{K}$. Dipyridamole reduces urinary albumin excretion in diabetic patients with normo- or microalbuminuria. Clin Nephrol. 1990; 33:130-135.

18. Walker RG, Yu SH, Owen JE, Kincaid-Smith P. The treatment of mesangial IgA nephropathy with cyclophosphamide, dipyridamole and warfarin: a two-year prospective trial. Clin Nephrol. 1990; 34:103-107.

19. Harmankaya O, Baştürk T, Oztürk Y, Karabiber N, Obek A. Effect of acetylsalicylic acid and dipyridamole in primary membranoproliferative glomerulonephritis type I. Int Urol Nephrol. 2001; 33:583-587.

20. Austin PC. A critical appraisal of propensity-score matching in the medical literature between 1996 and 2003. Statist Med. 2008; 27:2037-2049.

21. Mills DC, Smith JB. The influence on platelet aggregation of drugs that affect the accumulation of adenosine 3': 5 '-cyclic monophosphate in platelets. Biochem J. 1971; 121:185-196. 
22. Schoeffter P, Lugnier C, Demesy-Waeldele F, Stoclet JC. Role of cyclic AMP- and cyclic GMP-phosphodiesterases in the control of cyclic nucleotide levels and smooth muscle tone in rat isolated aorta. A study with selective inhibitors. Biochem Pharmacol. 1987; 36:3965-3972.

23. Blass KE, Block HU, Förster W, Pönicke K. Dipyridamole: a potent stimulator of prostacyclin $\left(\mathrm{PGI}_{2}\right.$ ) biosynthesis. Br J Pharmacol. 1980; 68:71-73.

24. Hewitson TD, Tait MG, Kelynack KJ, Martic M, Becker GJ. Dipyridamole inhibits in vitro renal fibroblast proliferation and collagen synthesis. J Lab Clin Med. 2002; 140:199-208.

25. Onozato ML, Tojo A, Goto A, Fujita T. Effect of combination therapy with dipyridamole and quinapril in diabetic nephropathy. Diabetes Res Clin Pract. 2003; 59:83-92.

26. Buranakarl C, Kitjtawonrat A, Pondeenana S, Sunyasujaree B, Kanchanapangka S, Chaiyabutr N, Bovee KC. Comparison of dipyridamole and fosinopril on renal progression in nephrectomized rats. Nephrology (Carlton). 2003; 8:80-91.

27. Taji Y, Kuwahara T, Shikata S, Morimoto T. Meta-analysis of antiplatelet therapy for IgA nephropathy. Clin Exp Nephrol. 2006; 10:268-273.

28. Liu XJ, Geng YQ, Xin SN, Huang GM, Tu XW, Ding ZR, Chen XM. Antithrombotic drug therapy for IgA nephropathy: a meta analysis of randomized controlled trials. Intern Med. 2011; 50:2503-2510.

29. Woo KT, Edmondson RP, Yap HK, Wu AY, Chiang GS, Lee EJ, Pwee HS, Lim CH. Effects of triple therapy on the progression of mesangial proliferative glomerulonephritis. Clin Nephrol. 1987; 27:56-64.

30. Zäuner I, Böhler J, Braun N, Grupp C, Heering P, Schollmeyer P. Effect of aspirin and dipyridamole on proteinuria in idiopathic membranoproliferative glomerulonephritis: a multicentre prospective clinical trial. Collaborative Glomerulonephritis Therapy Study Group (CGTS). Nephrol Dial Transplant. 1994; 9:619-622.
31. Hung CC, Yang ML, Lin MY, Lin HY, Lim LM, Kuo HT, Hwang SJ, Tsai JC, Chen HC. Dipyridamole treatment is associated with improved renal outcome and patient survival in advanced chronic kidney disease. Kaohsiung $\mathbf{J}$ Med Sci. 2014; 30:599-607.

32. Benson K, Hartz AJ. A comparison of observational studies and randomized, controlled trials. N Engl J Med. 2000; 342:1878-1886.

33. Concato J, Shah N, Horwitz RI. Randomized, controlled trials, observational studies, and the hierarchy of research designs. N Engl J Med. 2000; 342:1887-1892.

34. Cheng TM. Taiwan's new national health insurance program: genesis and experience so far. Health Aff (Millwood). 2003; 22:61-76.

35. Kuo KL, Hung SC, Liu JS, Chang YK, Hsu CC, Tarng DC. Iron supplementation associates with low mortality in predialyzed advanced chronic kidney disease patients receiving erythropoiesis-stimulating agents: a nationwide database analysis. Nephrol Dial Transplant. 2015; 30:1518-1525.

36. Hwang SJ, Yang WC, Lin MY, Mau LW, Chen HC; Taiwan Society of Nephrology. Impact of the clinical conditions at dialysis initiation on mortality in incident haemodialysis patients: a national cohort study in Taiwan. Nephrol Dial Transplant. 2010; 25:2616-2624.

37. Charlson ME, Pompei P, Ales KL, MacKenzie CR. A new method of classifying prognostic comorbidity in longitudinal studies: Development and validation. J Chronic Dis. 1987 ; 40:373-383. 